$\begin{array}{clc} & \text { Abstracts } \\ \text { Brain, Behavior } & & \text { Published online: July 10, 2007 } \\ \text { and Evolution } & \text { Brain Behav Evol 2007;70:205-210 } 10.1159 / 000105462 & \end{array}$

\section{The 2007 Meeting of the J.B. Johnston Club and the Nineteenth Annual Karger Workshop}

The 2007 meeting of the J. B. Johnston Club and Karger Workshop will be held immediately before the annual meeting of the Society for Neuroscience on Thursday, November 1 (the Nineteenth Annual Karger Workshop) and Friday, November 2 (the regular JBJC meeting), 2007. This year's Karger Workshop, made possible by the continuing support of Karger Publishers, was organized by Hans Hofmann and Caroly Shumway and is entitled 'Neurobiological Lessons Learned from Comparative Studies: Evolutionary Forces Shaping Brain and Behavior.' The Workshop features six invited talks by speakers who will review ways in which comparative studies have generated ideas about the evolution of behavior and the nervous system in disparate vertebrate and invertebrate taxa. On the following day, the program for this twenty-seventh annual JBJC meeting will consist of twelve talks submitted by JBJC members and selected by the JBJC Program Committee (Catherine McCormick, Cliff Summers, and Andrew Iwaniuk) plus a presentation by this year's invited Karger Speaker, Dr. Louis Lefebvre. Both the Karger Workshop and the regular JBJC meeting will take place at the San Diego Marriott Gaslamp Quarter, San Diego, Calif. Additional information and the final schedule of talks will be mailed to JBJC members before the meeting, and will be posted on the JBJC web site (www.jbjclub.org).

\section{Karger Workshop: \\ Neurobiological Lessons Learned from Comparative Studies: Evolutionary Forces Shaping Brain and Behavior}

\section{Organized by: Hans Hofmann}

(University of Texas at Austin, USA) and Caroly Shumway

(Boston University and The Nature Conservancy, USA)

Speakers giving presentations at the 2007 Karger Workshop are listed below. The final schedule of talks will be sent to the membership prior to the meeting and will be available at the registration desk during the meeting.

\section{Evolutionary Neural Links between Invertebrates and Vertebrates} (USA)

Linda Holland, Scripps-University of California San Diego

How Social and Ecological Forces Affect Mushroom Bodies

Sarah Farris, West Virginia University (USA)

How Social and Ecological Forces Affect Cichlid Brains

Caroly Shumway, Boston University and The Nature Conservancy (USA) and Hans Hofmann, University of Texas at Austin (USA)

\author{
Design Principles in Neural Circuits: Lessons from Birds and \\ Mammals \\ Sam Wang, Princeton University (USA)
}

Exploring the Origins of the Human Brain through Molecular Evolution

Eric Vallender, Harvard Medical School (USA)

Behavioral Flexibility and Brain Evolution in Birds

Louis Lefebvre, McGill University (USA)

\section{J.B. Johnston Meeting Abstracts}

Abstracts for talks scheduled for the 2007 annual meeting of the J.B. Johnston Club are listed in alphabetical order by presenting author. The final schedule of talks will be sent to the membership prior to the meeting and will be available at the registration desk during the meeting. This year's Karger Invited Speaker will be Louis Lefebvre. Dr. Lefebvre's abstract is listed first.

\section{Behavioral Drive and the Evolution of Enlarged Brains \\ Louis Lefebvre $^{\mathrm{a}}$, Zoltan Barta ${ }^{\mathrm{b}}{ }^{\text {D Daniel Sol }}{ }^{\mathrm{c}}$ \\ aDepartment of Biology, McGill University, Montréal, Québec (Canada); ${ }^{b}$ Behavioural Ecology Research Group, Department of Evolutionary Zoology, University of Debrecen (Hungary); ${ }^{\mathrm{C} C R E A F}$, Autonomous University of Barcelona, Catalonia (Spain) (louis.lefebvre@mcgill.ca)}

Brain enlargement in particular clades is presumed to have evolved in part because it provides fitness advantages in specific conditions. To support this idea, we must (1) demonstrate differences in survival or reproduction associated with brain size differences; (2) identify the mechanisms by which fitness differences are achieved; and (3) identify the processes by which brain enlargement has evolved in some clades, but not others.

Based on comparative analyses of birds and on simulations, we (1) identify differences in mortality (in 236 species) and colonization success (in 196 species) that correlate with brain size; (2) show that differences in innovativeness (mostly at the parvorder level) might be the key cognitive mechanism behind fitness differences; and (3) that behavioral drive can contribute to faster evolutionary diversification, increasing the proportion of large brained species over time.

Behavioral drive is the combined effect of innovation and social learning on encounter rates with possible new selection pressures. It is thought to be more frequent in larger-brained animals and to favor behavioral, and then genetic, divergence of sub-populations that have adopted different innovations. Comparative evidence

\begin{tabular}{ll}
\hline KARGER & ( ) 2007 S. Karger AG, Basel \\
Fax +41 61 306 1234 $34-8977 / 07 / 0703-0205 \$ 23.50 / 0$ \\
$\begin{array}{l}\text { E-Mail karger@karger.ch } \\
\text { www.karger.com }\end{array}$ & $\begin{array}{l}\text { Accessible online at: } \\
\text { www.karger.com/bbe }\end{array}$
\end{tabular}


suggests a higher rate of species and subspecies diversification (but not extinction) in larger-brained clades. Simulations show that moderate rates of innovation and intermediate to high rates of social learning yield the fastest diversification rates. An enlarged brain should thus be seen both as a product and a possible accelerator of evolution.

\section{Socially-Relevant Stimuli Modulate Cell Proliferation in the Adult Green Treefrog Brain (Hyla cinerea)}

\section{L.M. Almli}

Institute for Neuroscience, University of Texas, Austin, TX (USA) (lalmli@mail.utexas.edu)

Social experience plays an important role in regulating the neural, physiological, and hormonal changes that accompany the expression of reproductive behavior in vertebrates. Because acoustic communication is central to the reproductive behavior of amphibians, we sought to determine whether the acoustic cues that regulate behavior, physiology, and hormonal responses in a social context also regulate cell proliferation in the brain of adult green treefrogs (Hyla cinerea). In adult $H$. cinerea, cell proliferation zones are found throughout the forebrain, and in particular in the pallium, septum, striatum/nucleus accumbens, and the ventral hypothalamus (VH) [Almli and Wilczynski, 2007]. In this study, acoustic stimulus presentations, hormonal manipulations, and neuroanatomical methods were used to determine whether: (1) cell proliferation is affected by acoustic social cues; and (2) gonadal hormones are involved in socially-modulated cell proliferation. Adult H. cinerea were injected with 5-bromo-2' -deoxyuridine (BrdU; $100 \mathrm{mg} / \mathrm{kg}$, i.p.), a marker incorporated into actively dividing progenitor cells, on days 1,5 , and 9 of 10 days of nightly acoustic stimulus exposure: a recording of a natural chorus ('chorus') or random pure tones ('tones'). Brain sections were then processed using fluorescent immunohistochemistry for BrdU, and the blood plasma assayed for gonadal hormone levels (testosterone for males and estrogen for females). During the breeding season, frogs hearing their conspecific chorus had increased numbers of BrdU-immunopositive $(\mathrm{BrdU}+)$ cells in the preoptic area (POA) compared to tone controls $(\mathrm{p}<0.05)$. Cell proliferation declined overall as the breeding season progressed. In males, plasma testosterone levels were positively correlated with the number of proliferating POA cells, whereas no relationship between estrogen and proliferating POA cells was detected in females. Preliminary data suggest that cell proliferation in the $\mathrm{VH}$ is also socially-modulated and might exhibit a seasonal pattern similar to that seen in the POA. Future studies will investigate this phenomenon in other brain regions that exhibit naturally occurring adult cell proliferation. These results indicate that socially-relevant cues increase cell proliferation in brain regions mediating sexual behavior and endocrine regulation and might be modulated by testosterone levels in males.

Supported by NIH R01 MH057066 to Walter Wilczynski.

\section{Reference}

Almli LM, Wilczynski W (2007) Regional distribution and migration of proliferating cell populations in the adult brain of Hyla cinerea (Anura, Amphibia). Brain Res, in press.

\section{Visual Communication Changes Behavior Performances, but Not Reproductive or Stress-Relevant Gene Expression in a Teleost}

Chun-Chun Chen, Russell D. Fernald

Neurosciences Program and Department of Biological Sciences, Stanford University, Stanford, CA (USA)

(purepure@stanford.edu)

Social control of maturation is fairly common in fishes, yet the sensory cues mediating control are not well understood. We studied male cichlid fish, Astatotiapia burtoni which exist in two distinct and reversible social phenotypes: territorial ( $\mathrm{T}$ ) males that are reproductively capable and non-territorial (NT) that have regressed gonads and school with females. Interactions between mature males suggested that visual cues might be responsible for regulating male behavior and physiology. Here we asked whether visual cues alone could suppress male agonistic behavior, reproductive capacity or stress response.

Previously we showed that 1-day social suppression by intact physical contact increased cortisol and gonadotropin-releasing hormone $(\mathrm{GnRH})$ gene expression, but decreased testosterone level. Using a novel paradigm, we investigated whether visual cues alone could suppress a competitor male fish three times larger than a subject fish. We found that visual encounters with a much larger male alone can change an animal's behavior for at least one week. The observer tends to flee, school, and lose its dominant coloration during viewing. After a 1-day exposure, the smaller males increase $11-\mathrm{KT}$ level in plasma but do not activate GnRH or corticotropinreleasing factor gene expression, or cognate receptor gene expression in the pituitary. However, after three days of stimulation, viewers increase the total expression level of reproductive and stress related genes, but these transient increases did not affect the final hormone level. Furthermore, gene expression, circulation hormone levels, and gonad size show no difference from the control group after 7 days.

Clearly visual cues alone can stimulate a behavioral response but not a physiological change in the reproductive system or stress response. These data suggest that vision might facilitate social control of other males but full reproductive inhibition depends on additional cues.

\section{Dopamine Binds to Alpha2-Adrenergic Receptors: Evidence from Binding, Electrophysiological and Behavioral Studies}

C.A. Cornil ${ }^{\mathrm{a}, \mathrm{b}}$, G.F. Ball ${ }^{\mathrm{a}}$

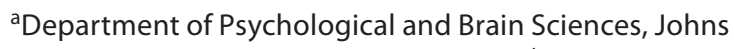
Hopkins University, Baltimore MD (USA); ${ }^{b}$ Center for Cellular and Molecular Neurobiology, University of Liège, Liège (Belgium) (Charlotte.cornil@ulg.ac.be)

The common view is that dopamine actions are mediated through dopaminergic receptors. However, an interaction with adrenergic receptors and transporters has also been suggested. Indeed, we have shown that in Japanese quail DA modulates the neuronal firing of medial preoptic neurons partly via its action on $\alpha_{2}$-adrenergic receptors. In order to characterize further this interaction and 
assess its existence in vivo, competition binding experiments were performed to investigate whether DA could displace the binding of a specific $\alpha_{2}$-adrenergic antagonist, $\left[{ }^{3} \mathrm{H}\right] \mathrm{RX} 821002$, utilizing autoradiographic procedures. A first set of competitions was performed on slices sectioned from minced and molded tissue prepared from male or female quail, zebra finch or rat brain. Each section was incubated in $5 \mathrm{nM}\left[{ }^{3} \mathrm{H}\right] \mathrm{RX} 821002$ in the presence of various concentrations of several unlabeled adrenergic and dopaminergic compounds. RX821002 binding displays a similar pharmacology in each species. Concentration-response curves indicate that DA binds to $\alpha_{2}$-adrenergic receptors at concentrations 10 - to 28 -fold higher than effective concentrations of norepinephrine (NE). The comparison of the $K$ i revealed no sex difference but a species difference mainly explained by the higher $\mathrm{Ki}$ of zebra finches relative to quail and rats. A follow-up study demonstrated that DA also binds human $\alpha_{2 \mathrm{~A}}$-adrenergic receptors expressed in S $f 9$ cells with an affinity 3-fold lower than NE. Because these experiments were performed on brain homogenates, it is not known where this interaction occurs in vivo. To address this issue, binding competitions were performed on quail and rat brain sections utilizing the same method. Based on concentration-response curves obtained in homogenates, only one concentration was used for each catecholamine $\left(10^{-7} \mathrm{M}\right.$ for $\mathrm{NE}$ and $10^{-6} \mathrm{M}$ for DA) in order to reduce $\left[{ }^{3} \mathrm{H}\right] \mathrm{RX} 821002$ binding to about $50 \%$. Areas analyzed comprised a variety of limbic regions such the preoptic area, the ventromedial nucleus of the hypothalamus, the bed nucleus of the stria terminalis (BST), the amygdala and the striatum. Male and female brains were compared. No sex significant difference was found with the exception of the rostro-medial part of the BST. In both species, DA and NE significantly reduced RX821002 binding in all areas analyzed. In rats, both NE and DA produced an equivalent marked reduction in binding (60 to $85 \%$ depending on the area considered). In quail, in contrast, NE produced a greater reduction (60 to $85 \%$ ) than DA (16 to $49 \%$ ). Together, these data provide evidence that DA not only acts on well-characterized dopaminergic receptors but also on adrenergic receptors and that this interaction occurs in avian as well as in mammalian species. The interaction of DA with adrenergic receptors does not seem anatomically specific but that there might be species differences in DA affinity for $\alpha_{2}$-adrenergic receptors relative to NE. Overall, these results are consistent with previous electrophysiological and behavioral studies supporting a physiological role for DA acting on adrenergic receptors. In particular, evidence suggests that this action of DA on adrenergic receptors might be involved in the control of male sexual behavior.

Supported by grant R01 NIH/MH50388. CAC was a BAEF Postdoctoral Fellow and is currently a F.N.R.S. Post-doctoral Researcher.

\section{The Key Deletion of an Adaptive Neural Circuit in Decapod Crustaceans}

\section{Zen Faulkes}

Department of Biology, The University of Texas-Pan American, Edinburg, TX (USA) (zfaulkes@utpa.edu)

Key innovations are evolutionarily novel features that are thought to drive diversification. Key deletions are a complimentary concept, which suggest that the evolutionary loss of a feature can also drive diversification. A potential example of a key deletion is the loss of escape responses of decapod crustaceans. Decapod crustacean escape responses are classic examples of adaptive, natural behaviors whose neural bases are well understood. In crayfish and clawed lobsters (Astacidea), the neural circuit responsible for escape responses is largely composed of giant neurons. Lateral giant interneurons (LGs) respond to posterior stimuli by generating a somersaulting tailflip; medial giant interneurons (MGs) respond to anterior stimuli with a backwards tailflip. Both sets of giant interneurons make monosynaptic connections to giant fast flexor motor neurons (MoGs), but with different patterns of connectivity, which largely explains the different kinematic patterns of tailflips. In shrimps and prawns (Dendrobranchiata, Caridea, and Stenopodidia), the giant interneurons are myelinated, and consequently have the highest conduction velocity known in any animal. The common hypothesis for most features of the escape circuit (e.g., giant neurons) is that there is strong selective pressure for individuals to respond to potentially threatening stimuli in the shortest possible time. Despite the apparent adaptive advantages of having a dedicated fast escape circuit, the escape circuit has been lost multiple times in reptantian decapods. Myelin is absent in the five reptantian infraorders (Palinura, Astacidea, Thalassinidea, Anomura, Brachyura). Palinuran spiny lobsters and slipper lobsters lack all giant neurons. This is particularly surprising given the morphological and ecological similarities of spiny lobsters to clawed lobsters, which have the escape circuit. Thalassinidean mud shrimp and anomuran hermit crabs lack the LGs. Other anomurans, including galatheoidean squat lobsters and hippoid sand crabs, lack the entire escape circuit, as do brachyuran crabs. Importantly, these taxa lacking the escape circuit are often speciose and widely distributed, not minor relictual taxa. The loss of the escape circuit might be a key deletion that promoted the radiation of some decapod crustacean lineages. The initial loss of the escape circuit may be a rare case of disaptation, resulting in species that are less well adapted than related species (i.e., those with the escape circuit). If the initial loss is tolerated, the resulting increased selection pressure for species to evolve new anti-predator strategies might have driven diversification. Additionally, the loss of the escape circuit might have removed constraints against change.

\section{Mapping of Neuronal Subpopulations in the Appendicularian Oikopleura dioica, with Some Comparative Considerations}

Joel C. Glover, Anne Mette Søviknes

Sars International Centre for Marine Molecular Biology, Bergen, Department of Physiology, University of Oslo (Norway)

(joel.glover@medisin.uio.no)

Increasing efforts using modern techniques to elucidate the structure and development of protochordate nervous systems have recently encompassed the appendicularians, largely as a result of genomic and genetic analyses of the species Oikopleura dioica. The CNS of this tunicate contains on the order of 100-120 neurons, and thus provides a model for the study of potential evolutionary constraints on the maintenance of chordate neural networks in the face of CNS miniaturization. We have used immunohistochemistry and in situ hybridization to identify and characterize neuronal subpopulations on the basis of neurotransmitter phenotype. This has led to the identification of putative cholinergic, GABAergic, and catecholaminergic neurons, and a description of their development from embryonic to adult stages. We have also described the development of neuromuscular projections and are beginning to assess central 
axon projection patterns and synaptic connections. A comparison of salient organizational features between the CNS of Oikopleura and other protochordates and vertebrates offers some insight into the diversification and maintenance of the chordate brain plan.

\section{Morphogenesis of the Cerebellum in Teleost Fish}

Yuji Ishikawa a Takako Yasuda ${ }^{\text {a }}$,Takahiro Kage ${ }^{\mathrm{a}, \mathrm{b}}$, Naoyuki Yamamoto d, Masami Yoshimoto ${ }^{c}$, Hiroyuki Takeda ${ }^{\text {b, }}$ Hironobu lto ${ }^{\mathrm{c}}$

aNational Institute of Radiological Sciences, Chiba, ${ }^{\text {b}}$ Department of Biological Sciences, Graduate School of Science, University of Tokyo, 'Department of Anatomy and Neurobiology, Nippon Medical School, Tokyo, dLaboratory for Fish Biology, Graduate School of Bioagricultural Sciences, Nagoya University, Nagoya (Japan) (ishikawa@nirs.go.jp)

Although the overall organization of the brains of teleost fish is generally similar to that of other vertebrates, there are several marked differences as well. One such difference is the cerebellum, which continues rostrally into the midbrain ventricle (the valvula cerebelli) only in actinopterygian (ray-finned) fishes. To analyze ontogenetic processes that underlie this remarkable morphological difference we have examined the embryonic development of the cerebellum of a teleost, medaka (Oryzias latipes), by histology and in situ hybridization using two gene (wnt1 and $f g f 8$ ) probes. The results were compared with previous studies in other vertebrates, particularly mammals. Paired cerebellar primordia were formed from the dorsal parts of the $f g f 8$-positive rostral metencephalon (isthmic region) and the fgf8-negative caudal metencephalon in the medaka neural tube. The rostralmost part of the metencephalon (prospective valvula cerebelli) protruded rostrally into the mesencephalic ventricle bypassing the morphological isthmic constriction during the development of the neural tube. The cerebellum was formed in the following successive stages: (1) enlargement of the cerebellar primordia and ventricle, (2) midline fusion of the left and right halves of the cerebellar primordia (3) formation of the secondary cerebellar matrices in the primitive cerebellum, and (4) growth and differentiation of the cerebellum. Our results show that the development of cerebellum is remarkably different in teleosts compared to mammals. Both rostral and caudal metencephalon develop into the cerebellum in medaka fish, whereas in mammals the rostral metencephalon (isthmic region) is reduced to a thin membrane (velum medullare anterius) during development and only the caudal metencephalon develops into the cerebellum.

\section{Exploring the Origin of Vertebrate Hearing: Neural Mechanisms of Directional Sensitivity and Frequency Selectivity in an Ancient Fish}

\author{
Michaela Meyer ${ }^{\mathrm{a}}$, Arthur N. Popper ${ }^{\mathrm{a}}$, Richard R. Fay ${ }^{\mathrm{b}}$ \\ aDepartment of Biology, University of Maryland, College \\ Park, MD, bParmly Hearing Inst., Loyola University of Chicago, \\ Chicago, IL (USA) (meyerm@umd.edu)
}

All animals need to gather information about their environment (for example, predator and prey detection) in order to survive. To reconstruct an acoustic view (or scene) of the environment based on sound, an animal needs to be able to detect, localize, discriminate, and identify sound sources. Because analysis of the auditory scene is fundamental to hearing, it has been hypothesized that the basic hearing capabilities used for scene analysis evolved early in vertebrate evolution and have remained relatively unchanged since then. Therefore, we hypothesize that the sound localization capabilities of non-teleost (often called 'ancient') bony fish such as sturgeon are similar to those of modern bony fish such as goldfish. The ears of non-teleost bony fishes are generally similar to those of other vertebrates except that they differ from teleosts in that the saccule and lagena are located in a single ventral pouch. This pouch contains a pasty otoconial mass instead of the dense otolith found in all teleosts. Despite these differences, responses in the eighth nerve show similar directional sensitivity and tuning to that shown in the teleost goldfish. We investigated the peripheral coding mechanisms for processing sound direction and frequency in the sturgeon. Single units were recorded extracellularly from the eighth nerve innervating saccule and lagena during stimulation with a three-axis shaker system. This system generated linear translational motion along various axes in the horizontal and mid-saggital planes of the fish, thereby simulating acoustic particle motion. To test frequency responses, displacements were generated in the vertical plane and the frequency varied between 50 and $1000 \mathrm{~Hz}$. Most units had cosineshaped directional response profiles in the horizontal and mid-sagittal planes. Saccular or lagenar afferents responded best to stimulus angles in the vertical plane at $90 \pm 20$ degrees, corresponding to sound sources directly from above or below the fish. The majority of afferents did not respond to stimuli in the horizontal plane. Such responses appeared to be correlated with the vertical orientation of the sensory epithelium and dorso-ventral orientation of hair cells on the epithelia that are innervated by these neurons. Best frequencies of afferents ranged between 100 and $300 \mathrm{~Hz}$. Thus similar neural codes for sound direction and frequency are found in the periphery of modern and ancient bony fishes, thereby supporting the hypothesis that basic auditory functions arose very early in vertebrate history. These data also suggest a difference in the encoding of sound direction between ancient and modern fishes. Species of both groups potentially receive information about sound direction from just one ear. The teleost brain might receive additional auditory information from differences in response between the right and left ears. This does not, however, appear to be the case in the more ancient sturgeon due to a nearly parallel orientation of the saccules and lagenae of the two ears. Thus directional hearing might have been further refined in teleosts when compared to the more ancient species.

\section{Telencephalic Organization in Lungfishes: A New Histochemical Model}

\section{R. Glenn Northcutt}

Department Neurosciences, UCSD, La Jolla, CA (USA)

(rgnorthcutt@ucsd.edu)

There is a dramatic accumulation of data on telencephalic organization in ray-finned fishes, but this is not the case for lobe-finned fishes. Dipnoans (lungfishes) represent the only feasible source of experimental data on this group, but unfortunately, there is little agreement on the number and extent of their telencephalic cell groups. Most differences in interpretation stem from disagree- 
ments regarding the position of the medial pallial - subpallial border, the extent of the dorsal and ventral striatopallidal systems, and the organization of the amygdala. There are presently two claims regarding the homology of the medial telencephalic wall: (1) Proponents of a restricted pallial model believe that the entire medial wall is homologous to the septum in other vertebrates; and (2) proponents of an extended pallial model believe that the medial wall, like that in other vertebrates, consists of a dorsal medial pallium and a ventral septum. Traditionally, the ventrolateral telencephalic wall in lungfishes has been interpreted as a dorsal striatum, but no dorsal pallidum has been recognized. Furthermore, the entire ventral floor of the telencephalon has been interpreted as an olfactory tubercle (ventral striatum), but a ventral pallidum has not been recognized. Finally, almost every part of the lateral telencephalic wall in lungfishes has been interpreted as an amygdala in one study or another. In order to clarify telencephalic organization in lobedfinned fishes, a new histochemical model of telencephalic organization in the African spotted lungfish, Protoperus dolloi, has been generated, based on the distribution of acetylcholinesterase, enkephalin, the neurotensin-related hexapeptide LANT6, nicotinamide adenine dinucleotide phosphate disphorase, and substance P. The distribution of these substances indicates that lungfishes have an extended pallium, comprising dorsal, lateral, medial, and ventral divisions. Both dorsal and ventral striatopallidal systems can be recognized, with the caudal cell group previously termed the olfactory tubercular cortex actually representing the dorsal pallidum. Furthermore, the ventral striatopallidal system appears to be far more restricted, rostrocaudally, than in earlier models. Rostrally, the ventral pallium appears to be homologous to the lateral amygdala of tetrapods and lies immediately dorsal to the subpallial anterior amygdala, which caps the dorsal striatum. Caudally, the lateral amygdala and the dorsal striatum are replaced by two cell groups that appear to be homologous to the central and medial divisions, respectively, of the amygdala in tetrapods. This new model is presently being tested in a series of experimental hodological studies. If it is supported, then telencephalic organization in lungfishes is far more similar to that in amphibians than was previously suspected.

\section{Attention and the Cholinergic System in Turtles \\ Alice S. Powers \\ Department of Psychology, St. John's University, Jamaica, NY (USA) (powersa@stjohns.edu)}

Selective attention involves focusing resources on one aspect of the environment and ignoring others. Research on humans and other mammals implicates acetylcholine in selective attention. Very little work on nonmammals, however, has addressed the possibility of their possessing a cognitive process like attention. In fact, some might argue that animals with as simple a brain as a reptile would not be capable of selective attention. Recent work in my laboratory has suggested, however, that turtles (Chrysemys picta) are capable of selective attention and that the cholinergic system of turtles mediates this ability to select among stimuli. The results of two experiments implicate acetylcholine in selective attention. The first is negative patterning. In this task, turtles were reinforced for responding to two individual elements (in our experiments a solid red key or a black key with white stripes on it), but not reinforced for responding to the compound of those elements (in our case, white stripes on a red background). Turtles given this task learned to respond to the elements but not to the compound. It has been shown by others that adding irrelevant stimuli disrupts performance on this task by causing the animals to respond less to the positive stimuli and more to the negative one. In our studies, we investigated the effects of the cholinergic antagonist scopolamine $(6.4 \mathrm{mg} / \mathrm{kg})$ on negative patterning. Scopolamine, but not methyl scopolamine (which does not cross the blood-brain barrier), disrupted negative patterning relative to turtles given saline in the same way that is found when irrelevant stimuli are added. This finding suggested that scopolamine disrupts selective attention. The second task involved comparing the learning of a horizontalvertical discrimination in the presence and absence of irrelevant dimensions. The irrelevant dimensions were a blue and white checked pattern on the walls of the experimental chamber and green indoor-outdoor carpeting on the floor. Although the addition of these irrelevant dimensions had no effect on the learning performance of control turtles given saline, turtles given scopolamine showed deficits on the task in the presence of the irrelevant dimensions. Interestingly, if the turtles given scopolamine were first trained on the discrimination task without the inserts, subsequent introduction of the irrelevant dimensions had no effect on their performance. Thus, acetylcholine appears to be involved in selective attention during acquisition, but not once the task has been learned. Experiments now in progress are investigating the effects of lesions of the basal forebrain cholinergic area on acquisition and retention of this task. These results provide strong support for the notion that turtles use selective attention to choose the relevant stimuli in their environment and that acetylcholine is necessary for this selection.

\section{Orbit Orientation, Binocularity and Eye Size in Relation to Visual Regions of the Avian Brain}

\author{
Douglas R.W. Wylie a, Christopher P. Heesy ${ }^{\mathrm{b}}$, Margaret I. Hallc, \\ Andrew N. Iwaniuk ${ }^{a}$ \\ aDepartment of Psychology, University of Alberta, Edmonton, \\ AB (Canada); ${ }^{b}$ Department of Anatomy, Arizona College of \\ Osteopathic Medicine, 'Department of Biomedical Sciences, \\ Midwestern University, Glendale, AZ (USA) (dwylie@ualberta.ca)
}

Orbit orientation is correlated with binocular field overlap and the relative size of visual structures in mammals such that species with more convergently oriented orbits have greater areas of binocular visual field overlap and relatively larger visual regions in the brain. Here, we test whether a similar pattern of correlated evolution is present in birds. Using both conventional statistics and modern comparative methods, we tested whether the relative size of the Wulst and optic tectum ( $\mathrm{TeO}$ ) were significantly correlated with orbit orientation, binocular visual field overlap and eye size in birds using a large, multi-species data set. In addition, we tested whether relative Wulst and $\mathrm{TeO}$ volumes were correlated with axial length of the eye. The relative size of the Wulst was significantly correlated with orbit orientation such that birds with a relatively large Wulst had more convergently oriented orbits. This was not true, however, of $\mathrm{TeO}$; the relative size of the $\mathrm{TeO}$ was not significantly correlated with orbit orientation. Similar results were obtained in our analyses of binocular visual field overlap; relative Wulst volume 
is positively correlated with binocular visual field overlap, whereas relative $\mathrm{TeO}$ volume is not. Finally, relative Wulst and $\mathrm{TeO}$ volume were weakly correlated with relative axial length of the eye, but these were not corroborated by independent contrasts. Overall, our results indicate that relative Wulst volume reflects orbit orientation and possibly binocular visual field overlap, but not eye size. Several mitigating factors could, however, have affected these results including the functional organization of Wulst and $\mathrm{TeO}$, the contribution of eye movements to binocularity and retinal morphology.

\section{Variation in Cerebellar Foliation in Cartilaginous Fishes: Ecological and Behavioral Considerations}

\author{
K.E. Yopak a, b , L. Frank b
}

aLeigh Marine Laboratory, University of Auckland, Warkworth (New Zealand); ${ }^{b}$ UCSD Center for Scientific Computation in Imaging, La Jolla, CA (USA) (kyopak@ucsd.edu)

The widespread variation in brain size and complexity that is evident in cartilaginous fishes (sharks, batoids, and chimaeroids) is related to both phylogeny and ecology. In addition to variation in encephalization and the relative development of major brain areas, there exists marked variation in the foliation of the cerebellar corpus. Volumetric analyses of the cerebellum in various vertebrate groups are often confounded by foliation, a trait that varies particularly throughout the chondrichthyan taxa. A foliation index (1-5), quantifying structural complexity of the cerebellum, was created based on length, depth, and number of folds and applied to more than 70 species of chondrichthyans. Although variation in the folding of the cerebellar corpus is correlated to phylogeny, as basal groups have lower foliation indices, there is substantial variation within and across clades that does not track with phylogenetic relationships. This foliation index was supplemented by newly emerging methodologies using MRI (Magnetic resonance Imaging) technology to further characterize foliation and provide a more quantitative analysis. High-resolution T1-weighted MR images were acquired on a GE Signa 3T Clinical scanner with a home built radio-frequency coil using a 3D spoiled gradient echo sequence for brain samples in situ. The degree of foliation was measured by performing cortical flattening on segmented images of the brain and comparing the volume of the expanded brain to the true volume as measured directly from segmentation. Cerebellum size has strong allometric correlations, but there exists much interspecific variation with regards to corpus folding. Both shark and batoid species with an active lifestyle and complex primary habitat, such as Carcharhinus, Sphyrna, and Aetobatus, demonstrate a relatively increased overall brain size and high cerebellar foliation. Less-active benthic and benthopelagic species, such as Notorhinchus, Cephaloscyllium, and Dipturus, show relatively smaller brains and a smooth cerebellar corpus. Correlations suggest that cerebellar development reflects the dimensionality of the environment and/or agile prey capture in addition to phylogeny.

\section{Singing Fish in vitro: Electrophysiological and Neuroanatomical Correlates of Sonic Neuron Rhythmic Activity}

M.C. Zee, A.H. Bass

Department of Neurobiology and Behavior, Cornell University, Ithaca, NY (USA) (mcz8@cornell.edu)

Teleost fishes, like all major vertebrate groups, have representative members that generate sound communication signals. Their vocal pattern generator (VPG) provides the simplest preparation for investigating how the cellular properties of sonic neurons lead to the production of social, context-dependent vocalizations. In particular, midshipman fish (Porichthys notatus) have an extensive hindbrain-spinal VPG that directly establishes the temporal features (fundamental frequency and duration) of natural calls and contains sonic motoneurons (SMNs) whose firing rate is determined by nearby pacemaker neurons. Here, we tested the hypothesis that the electroresponsive properties of SMNs alone might contribute to the temporal properties of the VPG. We first examined the firing properties of SMNs using whole-cell electrophysiology in vitro $(500 \mu \mathrm{m}$ thick, horizontal slices of the isolated hindbrain-spinal region). SMNs were silent at rest but fired 2-6 action potentials at a peak firing rate of $300 \mathrm{~Hz}$ that quickly attenuated in response to a $200 \mathrm{~ms}$ depolarizing step. SMNs could however fire on every cycle of a suprathreshold, sinusoidal current for at least $1 \mathrm{~s}$ at their peak firing rate. We next examined the effect of the $\mathrm{K}^{+}$channel blocker 4 aminopyridine (4-AP), which blocks a transient A-type $\mathrm{K}^{+}$channel in many systems, on SMNs. Low concentrations of $\mathrm{K}^{+}$ channel blockers elicit fictive locomotion in the rat spinal cord in vitro as well as rhythmic bursting in synaptically isolated neurons in the stomatogastric ganglion. Bath application of $1 \mathrm{mM} 4-\mathrm{AP}$ induced spontaneous rhythmic activity of SMNs either in the form of sustained rhythmic spikes or more often, in the form of a persistent slow wave $(0.4 \mathrm{~Hz})$ of rhythmic bursts. Spikes within a burst had an average firing frequency of $40 \mathrm{~Hz}$. The onset of burst activity occurred within 2 min of 4-AP entry into the bath and was sometimes irreversible. 4-AP oscillations were rapidly and reversibly blocked by $1 \mu \mathrm{M}$ tetrodotoxin but were unaffected by synaptic transmission blockers. The 4-AP oscillations could thus be intrinsic to SMNs, although they might yet arise, at least in part, from other electrically coupled SMNs (injection of neurobiotin into a single SMN leads to labeling of numerous SMNs throughout the entire nucleus). In sum, the results so far support the hypothesis that SMNs play an active role in shaping the VPG's temporal firing properties. As will be discussed, these traits have so far not been observed for other vertebrate vocal neurons, although they are consistent with the properties of neurons in other pattern generating networks.

Supported by NRSA F32-DC007792 to MCZ, NIH (DC00092) and NSF (IBN 0516748) to AHB. 